\title{
How do limitations in spectrum fungibility impact spectrum trading?
}

\author{
Marcela Gomez, Martin BH Weiss \\ Graduate Telecommunications and Networking Program \\ School of Information Sciences \\ University of Pittsburgh \\ Pittsburgh, PA \\ Email: mmg62, mbw@ @itt.edu
}

\begin{abstract}
Secondary markets for spectrum trading have been considered an important solution for generating spectrum opportunities in an environment where scarcity is the rule. Nonetheless, an important factor when envisioning a successful spectrum trading environment is to consider how comparable an available frequency is to the frequency an spectrum user prefers. With this aim, we consider the fungibility scores previously determined in [1] in order to explore additional parameters that can influence this quantification of the level of fungibility. Further, we merge these fungibility calculations with an existing spectrum trading model, SPECTRAD [2], seeking to determine the actual impact of the limitations of spectrum fungibility in the market viability.
\end{abstract}

\section{INTRODUCTION}

Increasing communication needs and opportunities fueled by technology changes have resulted in the reduced availability of electromagnetic spectrum. Countless solutions to use spectrum more efficiently have been analyzed over time; among them have been secondary markets for electromagnetic spectrum. This solution was first mentioned by Coase in [3] and has also been considered in more recent work [4], [5], where secondary spectrum markets are seen as a means to make a more efficient use of this resource.

In an ideal spectrum trading scenario, electromagnetic spectrum would be considered an asset or commodity like other traded commodities. In such a world, we could deploy a spectrum trading system in which, given the perfect fungibility characteristics of spectrum, each seller would know that the spectrum he offers would satisfy the demands of every buyer and at the same time, each buyer would be confident about the compatibility between the spectrum market purchases and his specific needs.

Unfortunately, the physical characteristics of electromagnetic spectrum frequency are not consistent with this scenario. In fact, electromagnetic spectrum is not fungible in space nor frequency. That is, spectrum availability in one geographic region cannot satisfy demands in another region and the propagation of electromagnetic waves is a function of frequency, which means that one band is not necessarily fungible with another band. The limitations of spectrum fungibility was first examined in [1]. In this paper, a fungibility score was proposed as a means to establish a level of substitutability of two different frequencies.

In this paper, we extend the analysis of the parameters that would influence spectrum fungibility and consider their impact on the liquidity of (hypothetical) spectrum trading markets. We do this by refining the calculation of these fungibility scores, applying them to particular spectrum bands and using them as an input to simulated spectrum trading markets using Caicedo's SPECTRAD model [6] to determine the impact of fungibility on the viability of spectrum markets. In order to quantify the impact of the limitations in spectrum fungibility, we consider as a reference point the market viability results obtained in [6], which model the market characteristics and conditions for viability when considering a perfectly fungible electromagnetic spectrum.

This paper is structured as follows: section II presents the approach to calculating fungibility scores. Section III explains how the spectrum trading market has been modeled and the considerations 
made in order to incorporate non-perfect fungibility conditions. Section IV presents the market viability results under non-perfect fungibility conditions and further compares them to those obtained in [6]. Section V contains our conclusions and specifies research directions that will be considered in the future.

\section{FungibiLity SCORES}

An evident first step for determining the impact of the limitations in spectrum fungibility in a spectrum trading scenario is to develop a quantitative measure of a rather abstract notion, i.e., non-perfect fungibility. The use of fungibility scores have been proposed to achieve this [1], which could quantify the level of comparability between two frequencies (e.g., the preferred and available frequency bands). The authors of that paper considered the free-space propagation loss differences across electromagnetic frequencies to determine how substitutable a certain frequency band is with another. Their results represent the level of comparability of the different preferred frequencies and available spectrum options in the form of both a "distance" score and a "probabilistic" score. This leaves the spectrum buyer or requester with a measure to consider how applicable is a given frequency to his actual needs, and hopefully would make it easier to decide which frequency to opt for.

Free-space path loss sets an explicit difference in the characteristics and capabilities of various frequency bands. However, most actual transmissions and communications take place in geographic environments with characteristics very different from free-space. Additionally, infrastructure characteristics (such as antenna height) play an important role in the propagation conditions. It is for these reasons that we have delved deeper into the propagation loss analysis and considered alternate propagation models for calculating fungibility scores, considering, in particular, the empirical propagation loss models such as Okumura-Hata, Cost 231-Hata and Walfish Ikegami.

When using these models for determining fungibility scores, we can analyze the multi-dimensional characteristics of spectrum, and further articulate which are the modifications that a spectrum buyer, with a preferred frequency, would have to make to their current system in order to satisfy their traffic requirements with an alternate frequency. These modifications could include factors such as the distance from the base station, the base station antenna height, and the mobile antenna height. The most interesting part however, is how these modifications and propagation loss considerations result in perhaps more quantifiable conditions such as coverage and capacity. Thus, we can now shed more light onto the question of how opting for a new frequency affects the maximum capacity and coverage that can be achieved. More importantly, we can determine which specific change (i.e., increase/decrease of bandwidth, distance from the base station) that a spectrum user can consider in order to achieve appropriate (not to say equal) characteristics with an alternate, available frequency.

In this work, we will focus on the calculation of fungibility scores based on coverage and capacity considerations. For presenting these scores, we refine the approach used in [1]. We determine probabilistic and distance fungibility scores by comparing the results obtained with a reference frequency $f_{1}$ to those obtained with a frequency $f_{2}$. The probabilistic score, as shown in (1), corresponds to the ratio of the results obtained with $f_{1}$ and $f_{2}$, and the distance score, as shown in (2), corresponds to the Euclidean distance between the results obtained with the two different frequencies. Note that the ideal probabilistic score is 1 , and the ideal distance score is 0 . Both of these conditions imply that the two considered frequencies are perfectly fungible.

$$
\begin{gathered}
P_{\text {score }}=\min \left(\frac{f_{1}}{f_{2}}, 1\right) \\
D_{\text {score }}=\frac{\max \left(\left(d_{1}-d_{2}\right), 0\right)}{d_{1}}
\end{gathered}
$$

\section{A. Coverage Fungibility Score}

An important reason for spectrum users to seek further spectrum assets, besides fulfilling their traffic requirements, is to increase their coverage area. This would thus be a reason for them to enter the spectrum trading market and opt for additional spectrum bandwidth units. If we take into account the value of the minimum required received power, we can determine the maximum distance that we could cover. This maximum distance corresponds to the maximum cell radius and gives us a notion of the coverage radius that can be achieved with 
a given frequency. The received power can be estimated through equation (3), where $P_{t}$ is the transmit power, the transmitting and receiving antennae gains are $G_{t}$ and $G_{r}$ respectively, and $L_{p}$ accounts for the path loss. Since we are working with a specific minimum required received power, we utilize (3) to determine the corresponding maximum allowable path loss. Finally, through the empirical path loss models that we have utilized, we can determine the maximum distance associated with this path loss requirement, and thus, we will obtain the maximum cell radius or coverage radius.

In this manner, we compare the maximum cell radius for determining the coverage fungibility score for $f_{1}$ and that for $f_{2}$, given a common, minimum received power requirement. For the calculations presented here, we consider a transmitting power $P_{t}$ equal to $1 \mathrm{~mW}$ and the transmitting and receiving antennae gains are set to 1 . The minimum allowable received power is set to $-80 \mathrm{dBm}$.

$$
P_{r}=P_{t}(d B m)+G_{t}(d B)+G_{r}(d B)-L_{p}(d B)
$$

\section{B. Capacity Fungibility Score}

In addition to coverage, users may trade spectrum to increase their capacity in a given coverage area. In this case, the Shannon capacity formula (4) was applied to two different circumstances: when capacity is considered at a fixed distance from the transmitter, and when capacity is evaluated at the cell edge of the reference frequency.

$$
C=B \log _{2}(1+S N R)
$$

Capacity at a fixed distance: In this scenario, we consider the power received at a given reference distance for the SNR calculations (i.e., $1 \mathrm{Km}$ ) using equation (3). The noise is estimated using the noise temperature floor formula (5), or its equivalent in $\mathrm{dB}(6)$, where $F$ is the noise figure of the receiver, $k$ is the Boltzmann's constant, $T$ is the reference temperature $(290 \mathrm{~K})$ and $B$ is the considered bandwidth.

The reference value for this fungibility score will be the capacity achieved with a given frequency at the reference distance with a specific bandwidth (i.e., capacity achieved by $700 \mathrm{MHz}$ at $1 \mathrm{Km}$ with $1 \mathrm{MHz}$ of bandwidth). The compared value will be the capacity achieved at the same reference distance with different frequencies and varying bandwidths. The point at which the probabilistic score reaches 1 or the distance score reaches 0 , signals the amount of bandwidth of $f_{2}$ required to equal the performance of $f_{1}$.

$$
\begin{aligned}
N & =F k T B \\
& =F(d B)+k(d B m / H z / K)+T(d B m)+B(d B m)
\end{aligned}
$$

Capacity at the cell edge: In this scenario, we consider the maximum cell radius at which a reference frequency complies with the minimum received power requirements. In other words, the reference will be the capacity achieved with a certain frequency at its cell edge with a reference bandwidth. For the compared frequency, we calculate the capacity achieved at the distance equivalent to the cell edge of the reference frequency (not its own cell edge) with different values of bandwidth and the corresponding noise. Again, if we compare the capacities achieved with $f_{1}$ and $f_{2}$, through their ratio or the Euclidean distance separating them, we obtain a new capacity fungibility score. The interpretation of the score is the same as that of the capacity at a fixed distance scenario: the score corresponds to the required bandwidth of $f_{2}$ to match the capacity of $f_{1}$, but this time at the cell edge of the reference frequency.

\section{Fungibility Score Results}

The tables presented in what follows show the fungibility scores that we have obtained when applying the methods explained above. Table I shows the parameters that he have considered as a reference throughout our calculations and their corresponding values. We have chosen these values according to those specified in [2] in our aim to merge this results with the spectrum trading environment presented in that same work. As it can be observed, in this particular analysis we have a set of preferred frequencies which correspond to the reference frequencies and we consider only one available frequency, which in turn corresponds to the compared frequency.

Table II contains the coverage fungibility scores that we have obtained taking into account the foregoing reference and compared parameters. To complement these results, figure 1 portrays the coverage fungibility scores of the preferred frequencies when compared to frequencies ranging from $700 \mathrm{MHz}$ to $2000 \mathrm{MHz}$. 
As it can be observed in Table II, the distance scores are complementary to the probabilistic scores, which is the reason why we have included only one of the two scores in our figures.

\begin{tabular}{|c|c|}
\hline \multicolumn{2}{|c|}{ Reference Parameters } \\
\hline Parameter & Reference Value \\
\hline Preferred Frequencies & $\begin{array}{c}700,1000,1500,1700, \\
1900 \text { and } 2000 \mathrm{MHz}\end{array}$ \\
\hline Available Frequency & $1900 \mathrm{MHz}$ \\
\hline Bandwidth & $200 \mathrm{KHz}$ \\
\hline Distance & $1 \mathrm{Km}$ \\
\hline Transmitted Power & $1 \mathrm{~mW}$ \\
\hline $\begin{array}{l}\text { Minimum Allowable } \\
\text { Received Power }\end{array}$ & $-80 \mathrm{dBm}$ \\
\hline Base Station Height & $50 \mathrm{~m}$ \\
\hline Geographic Environment & Medium/Small city \\
\hline Mobile Antenna Height & $1 \mathrm{~m}$ \\
\hline Noise Figure & $0 \mathrm{~dB}$ \\
\hline Width of road & $20 \mathrm{~m}$ \\
\hline Buildings separation & $40 \mathrm{~m}$ \\
\hline Buildings height & $15 \mathrm{~m}$ \\
\hline Phi & 90 \\
\hline
\end{tabular}

TABLE I

REFERENCE PARAMETERS UTILIZED FOR THE CALCULATION OF FUNGIBILITY SCORES

Coverage Fungibility Scores

\begin{tabular}{ccc}
\hline \hline $\begin{array}{c}\text { Preferred } \\
\text { Frequency } \\
\text { [MHz] }\end{array}$ & $\begin{array}{c}\text { Distance } \\
\text { Score }\end{array}$ & $\begin{array}{c}\text { Probabilistic } \\
\text { Score }\end{array}$ \\
\hline & & \\
700 & 0.5766 & 0.4234 \\
1000 & 0.4368 & 0.5632 \\
1500 & 0.2000 & 0.8000 \\
1700 & 0.1040 & 0.8960 \\
1900 & 0 & 1 \\
2000 & 0 & 1 \\
\hline
\end{tabular}

TABLE II

Coverage Fungibility SCORES. THE PREFERRED FREQUENCIES ARE COMPARED TO AN AVAILABLE CENTER FREQUENCY OF $1900 \mathrm{MHZ}$
Following the coverage-based results, we present our capacity fungibility scores at $1 \mathrm{Km}$ (fixed distance) in Table III. The scores in this table quantify the level of comparability of $1900 \mathrm{MHz}$ with different values of bandwidth to the preferred frequency operating with $200 \mathrm{KHz}$ of bandwidth. It is in this manner that the bandwidth value corresponding to a distance/probabilistic score of $0 / 1$ respectively, indicates that with that bandwidth the available frequency can match the performance of the preferred frequency. In this scenario, we show that $1900 \mathrm{MHz}$ with $20 \mathrm{MHz}$ of bandwidth would not reach the perfect fungibility status when compared to 700 $\mathrm{MHz}$, while when compared to $2000 \mathrm{MHz}$, the reference bandwidth will suffice. Figure 2 supports the results presented in Table III and shows the capacity probabilistic scores for values of bandwidth in the range of $200 \mathrm{KHz}$ to $20 \mathrm{MHz}$.

Capacity Fungibility Score - At $1 \mathrm{Km}$

\begin{tabular}{|c|c|c|c|}
\hline $\begin{array}{c}\text { Preferred } \\
\text { Frequency } \\
{[\mathrm{MHz}]}\end{array}$ & $\begin{array}{c}\text { Bandwidth } \\
\text { (Available } \\
\text { Frequency) } \\
\text { [MHz] }\end{array}$ & $\begin{array}{c}\text { Distance } \\
\text { Score }\end{array}$ & $\begin{array}{c}\text { Probabilistic } \\
\text { Score }\end{array}$ \\
\hline 700 & $\begin{array}{c}0.2 \\
1 \\
20 \\
\end{array}$ & $\begin{array}{l}0.6552 \\
0.3856 \\
0.1968 \\
\end{array}$ & $\begin{array}{l}0.3448 \\
0.6144 \\
0.8032 \\
\end{array}$ \\
\hline 1000 & $\begin{array}{c}0.2 \\
1 \\
11.22 \\
\end{array}$ & $\begin{array}{c}0.5649 \\
0.2246 \\
0 \\
\end{array}$ & $\begin{array}{c}0.4351 \\
0.7754 \\
1 \\
\end{array}$ \\
\hline 1500 & $\begin{array}{c}0.2 \\
0.483\end{array}$ & $\begin{array}{c}0.3085 \\
0\end{array}$ & $\begin{array}{c}0.6915 \\
1 \\
\end{array}$ \\
\hline 1700 & $\begin{array}{l}0.2 \\
0.3\end{array}$ & $\begin{array}{c}0.1686 \\
1 \\
\end{array}$ & $\begin{array}{c}0.8314 \\
1 \\
\end{array}$ \\
\hline 1900 & 0.2 & 0 & 1 \\
\hline 2000 & 0.2 & 0 & 1 \\
\hline
\end{tabular}

TABLE III

RESULTING CAPACITY FUNGIBILITY SCORES AT 1 KM DISTANCE FROM THE TRANSMITTER. THE PREFERRED FREQUENCIES OPERATE AT $200 \mathrm{KHZ}$ OF BANDWIDTH AND ARE COMPARED TO AN AVAILABLE FREQUENCY OF $1900 \mathrm{MHZ}$.

Table IV includes the resulting capacity fungibility scores when analyzing the cell edge of the reference or preferred frequencies. In the same manner as the previous scenario, the preferred frequencies are operating at $200 \mathrm{KHz}$ of bandwidth and will be compared at their cell edge with the performance 


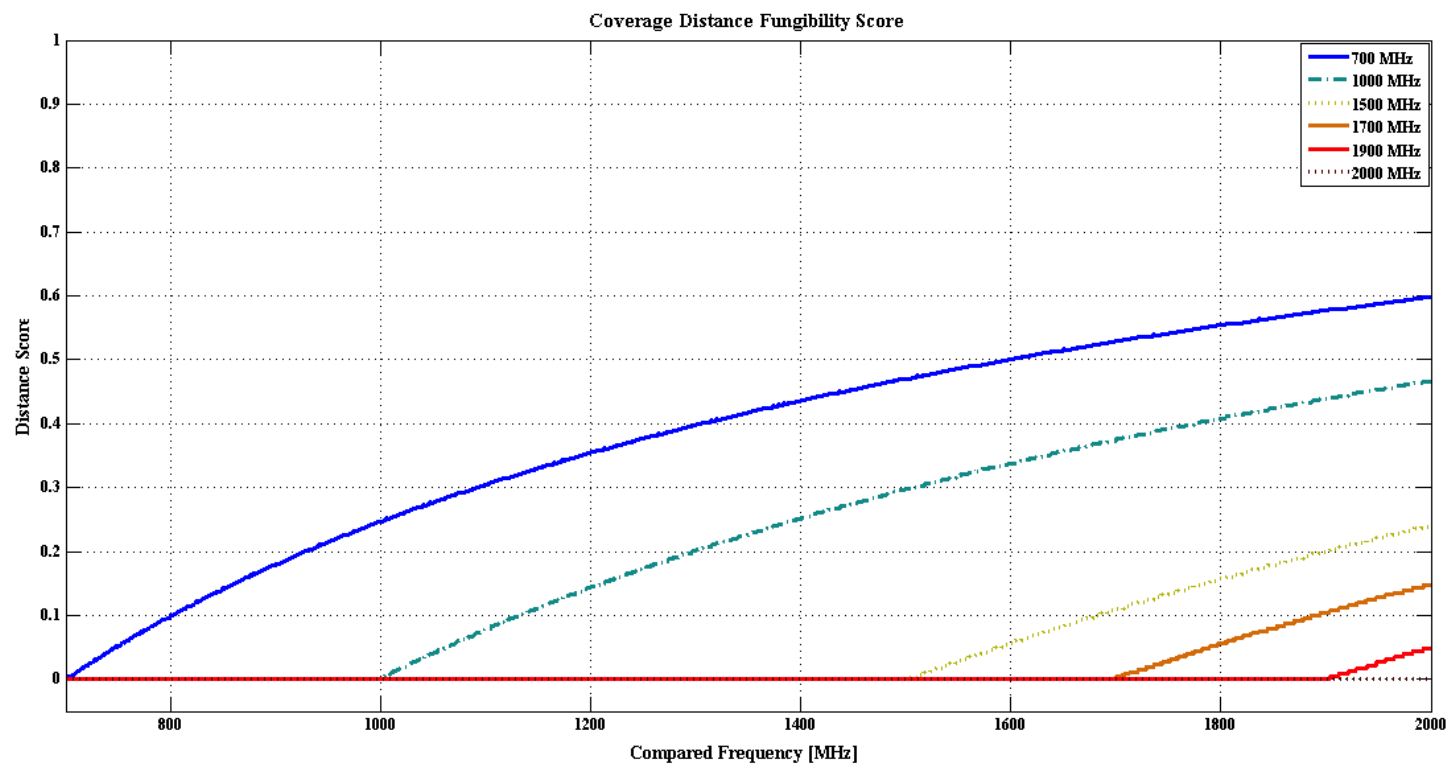

Fig. 1: Coverage Distance Score for the preferred frequencies when compared to frequencies in the $700-2000 \mathrm{MHz}$ range

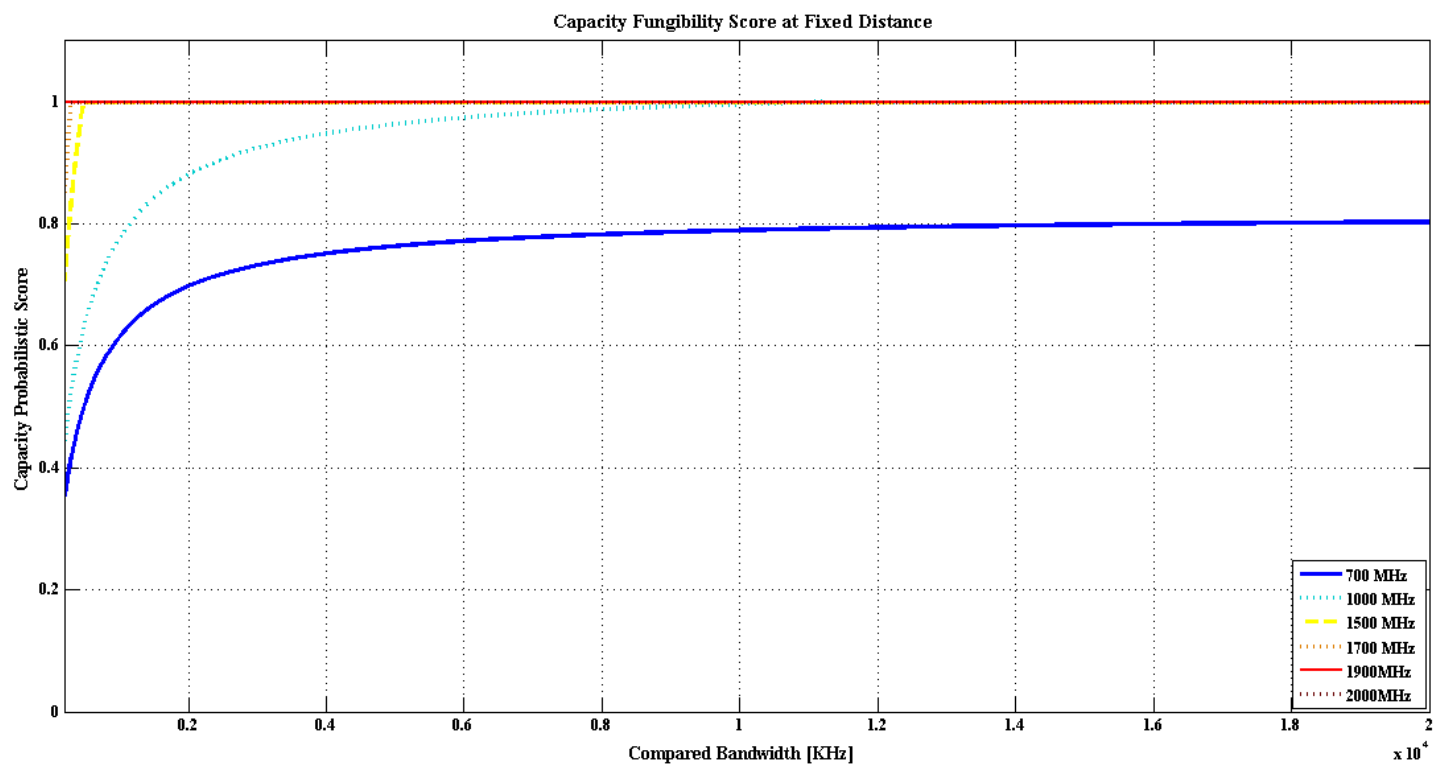

Fig. 2: Capacity probabilistic score comparing the preferred frequencies to $1900 \mathrm{MHz}$ at $1 \mathrm{Km}$ to portray the fixed distance scenario

of $1900 \mathrm{MHz}$ with different values of bandwidth. Figure 3 complements the results presented in Table IV for additional values of bandwidth. From these results we can point out that at the cell edge of the reference frequency, for our minimum received power requirements, the available frequency will require less bandwidth for achieving perfect fungibility scores. In fact, the required bandwidth is fairly close to the reference value.

\section{SPECTRUM TRADING}

After calculating fungibility scores, the next step is to incorporate this notion of non-perfect fungibility into the spectrum market model and determine the effect they have in the resulting market viability and liquidity. For this purpose, we use the spectrum market modeling tool SPECTRAD, presented in [6]. This tool models a secondary spectrum market in which spectrum users take part in continuous double 


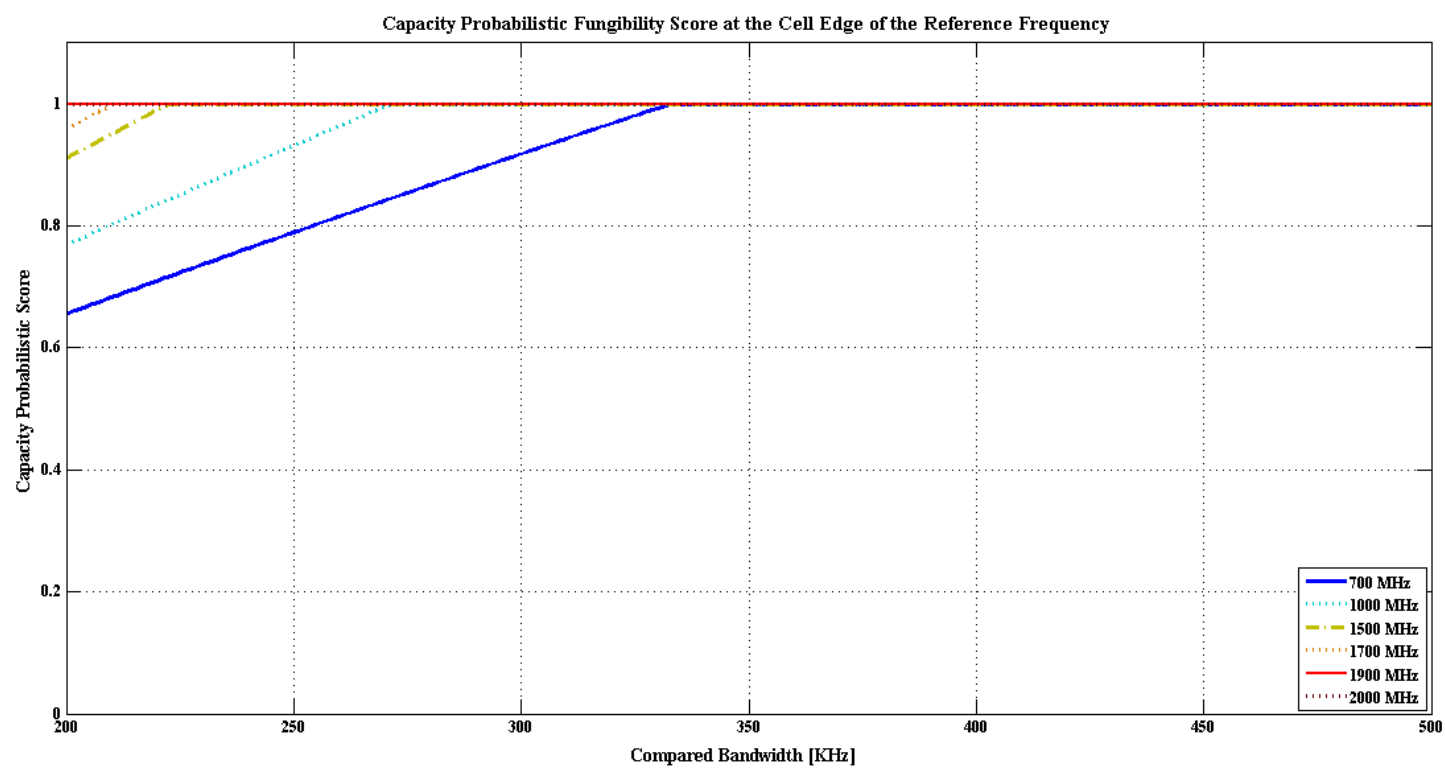

Fig. 3: Capacity probabilistic score comparing the preferred frequencies with $1900 \mathrm{MHz}$ at the cell edge of the preferred frequencies.

Capacity Fungibility Score - Cell Edge

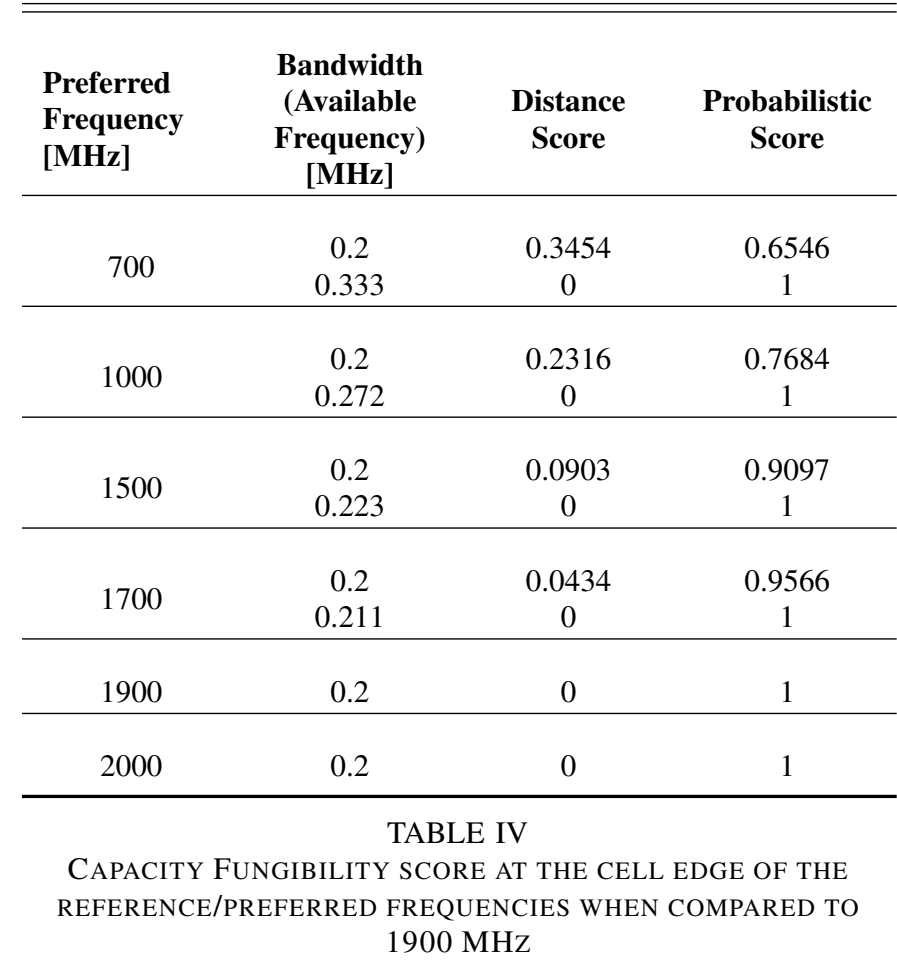

auctions in order to acquire a lease or a license for bandwidth units that are being offered in the market. The market participants in a spectrum-trading scenario are the Spectrum Exchange, Spectrum Users and the Regulator. The spectrum users can be thought of as users who hold spectrum and would desire to sell it: Spectrum License Holders (SLH) and also users who need to acquire bandwidth units in order to fulfill their traffic requirements, called Spectrum License Requestors (SLR). The spectrum market can be modeled in various ways, e.g., exchanges or brokers. Since our aim is to present a first approach into incorporating fungibility scores to a spectrum trading market, we will consider a spectrum exchange with Band Manager functionality. Further, in our study, this exchange will not act as a pooling point. According to [2], additional characteristics of this type of exchange are as follows:

- The trade-able spectrum is entirely held by the exchange,

- The exchange is in charge of providing the spectrum and this spectrum should return to the exchange upon cease of the leasing or sale agreement, and

- The exchange grants the corresponding authorizations for use of the spectrum, but it is not in charge of configuring any equipment for spectrum use.

The market viability results obtained in [2] represent the outcome of a spectrum trading market when spectrum is perfectly fungible. For this reason, we would like to use these results as a reference point to 
determine the conditions that become "harmful" for the market when considering non-perfect fungibility characteristics. With this in mind, we incorporated the fungibility criteria in the least intrusive manner possible into the Band Manager Exchange market modeled in SPECTRAD.

\section{A. Model Characteristics}

The characteristics of the model we have tested are as follows:

Market Participants: The spectrum exchange, in the form of a Band Manager (BM), holds a specific number of $200 \mathrm{KHz}$ bandwidth units of spectrum (BBU) in the $1900 \mathrm{MHz}$ band. The BM will offer its available assets for lease in each bid round and determine a cutoff price for the spectrum based on the bids of the spectrum buyers. At the end of the bidding rounds, the BM will grant the spectrum to the users with bids above the cutoff price.

The spectrum users will be limited to SLRs who are seeking a lease or license transfer of spectrum from the BM. Note that the SLRs' preferred frequency is not the same frequency band offered by the Band Manager; in this way, the SLRs will post their bids according to their valuation of the spectrum offered by the BM. Following [2], the maximum price an SLR is willing to pay for spectrum corresponds to the price the same SLR would pay for an AT unit. If at the end of the bidding rounds, an SLR is not able to obtain bandwidth units of spectrum, it will opt for alternate technology (AT) units to satisfy its requirements. As specified in [2] the AT units can be viewed as alternate equipment the user can utilize instead of the spectrum offered by the BM for fulfilling its traffic demands ${ }^{1}$. In this work we have assumed that an AT unit will have the same capabilities as the preferred frequency of an SLR.

The Regulator will maintain records that map bandwidth units of a frequency band to the current holders of that spectrum. In addition, the regulator has the responsibility of overseeing the legitimacy of the transactions taking place in the market; however, it will not take an active position in the market unless anomalies would require its action.

\footnotetext{
${ }^{1}$ Alternate technologies might include wireline systems, investments to make the existing spectrum more efficient, the use of unlicensed bands, etc.
}

User Valuation of Spectrum: One of the most important considerations for deploying secondary spectrum-trading markets is the goal of achieving a more efficient use of spectrum, which includes granting this resource to the users who value it the most. This would imply a highly efficient spectrum assignment method that considers market gains that cannot be determined during the initial spectrum assignment by the primary market [4]. When including fungibility considerations in the market, recall that users with a preferred center frequency $f_{1}$ will value an available BBU of frequency $f_{2}$ on the basis that it can satisfy its traffic and transmission requirements with it. In this manner, an SLR will determine a maximum price that it is willing to pay for the spectrum frequency, $f_{2}$, offered by the BM. The maximum price corresponds to the price the SLR would pay for AT units, which would also satisfy its requirements.

In the SPECTRAD model, the price of BBUs and AT units are related by (7). From this expression, we obtain the limit price that an SLR is willing to pay for a BBU, as shown in (8).

$$
\begin{gathered}
(\text { BBUnits })(\text { LimitPricePerBBU })= \\
(\text { ATUnits })(\text { PricePer } A T) \\
\text { LimitPricePerBBU }=\frac{\text { ATUnits }}{B \text { BUnits }}(\text { PricePer } A T)
\end{gathered}
$$

In the case of perfect fungibility the number of AT units would be the same as the number of BBUs, thus showing that the maximum price per BBU that a user would pay corresponds to the same price the user would pay for an AT. When considering our non-perfect fungibility scenario, this outcome will no longer occur. Since BBUs and AT units will provide different capacities, if we consider (9) and (10), we can determine that we will need different numbers of BBUs than we would need AT units. Moreover we can relate the two latter equations and obtain (11), which actually corresponds to the capacity probabilistic fungibility score we presented in the first part of this paper.

$$
\begin{aligned}
\text { ATunits } & =\frac{\text { TrafficToServe }}{\text { CapacityPerAT }} \\
B B U \text { nits } & =\frac{\text { TrafficToServe }}{\text { CapacityPerBBU }}
\end{aligned}
$$




$$
\frac{\text { ATUnits }}{\text { BBUnits }}=\frac{\text { CapacityPer } B B U}{\text { CapacityPer } A T}
$$

It is in this manner that we have introduced the non-perfect fungibility considerations in the economic valuation of spectrum from SLRs. To summarize, the maximum price that a user would pay for a bandwidth unit will now be limited by the ratio between the capacity it can reach with the offered bandwidth unit and the capacity it can reach with an AT, which has been calculated to match the capacity obtained when using the preferred frequency.

SPECTRAD considers three levels of user valuation of spectrum: low, medium and high. Provided the BM offers a center frequency of $1900 \mathrm{MHz}$ and based on the fungibility scores presented in the first part of this paper, we will consider that the users with a low valuation of spectrum have a preferred frequency of $700 \mathrm{MHz}$, the users with a medium valuation will have a preferred frequency of 1700 $\mathrm{MHz}$ and the users with a high spectrum valuations will be those with a preferred frequency of 2000 $\mathrm{MHz}$.

Relating the fungibility score to the monetary valuation of spectrum, we determine that the low, medium and high valuation users will have the limit BBU prices given by (12), (13), and (14) respectively.

$$
\begin{gathered}
\text { LimitPricePer } B B U=0.3448 * \text { PricePer } A T \\
\text { LimitPricePer } B B U=0.8314 * \text { PricePer } A T \\
\text { LimitPricePerBBU }=\text { PricePer } A T
\end{gathered}
$$

The price per AT has been determined following [2]. Thus, the range of prices for an AT unit according to the valuation level is defined through (15), (16), and (17). The users will be assigned a random value within this range according to their level of AT valuation. For our non-perfect fungibility case, we shall consider that SLRs' spectrum valuation is inversely proportional to their ATs' valuation. For instance, an SLR with a low spectrum valuation will be assigned AT prices from the PricePerAT high valuation range. In these expressions, minATPrice corresponds to the minimum price a user would pay for an AT unit and is equal to 100 monetary units; maxATPrice is the maximum price to pay for an AT unit and equals 250 monetary units; finally, rangePrices is defined by (18).

$$
\begin{array}{r}
\text { PricePerAT } T_{\text {low }}=\text { From }(\text { minATPrice }) \\
\text { To }(\text { minATPrice }+ \text { rangePrices }) \\
\text { PricePerAT } T_{\text {med }}=\text { From }(\text { minATPrice }+ \text { rangePrices }+1) \\
\text { To }(\text { minATPrice }+2(\text { rangePrices }))
\end{array}
$$

PricePerAT $T_{\text {high }}=$ From $($ minATPrice $+2($ rangePrices $)+1)$ To (maxATPrice $)$

$$
\text { rangePrices }=\frac{(\max A T \text { Price }- \text { minATPrice })}{3}
$$

Running Environment: The modifications that have been made to SPECTRAD, do not change the running considerations of the original model. SPECTRAD has been developed in Java and runs on top of REPAST Simphony, an agent-based modeling platform which provides a running environment appropriate for ACE models.

In [2], the market viability results obtained using SPECTRAD were the output of testing various scenarios given by the variation of the following parameters:

- Number of Market Participants

- Distribution of spectrum users' valuation level

- Available Spectrum

- Market Type

We have taken into account the same parameters and their variations to test our non-perfect fungibility compliant version of SPECTRAD. The values of these parameters are summarized in Table V. Note that, in order to thoroughly analyze the effect of non-perfect spectrum fungibility, we have started with a scenario in which all spectrum users have a low valuation of the spectrum. Following this, all SUs should have a $700 \mathrm{MHz}$ preferred center frequency. We shall consider this scenario, as the worst-case situation for non-perfect fungibility and market liquidity. 
SPECTRAD Parameters

\begin{tabular}{ll}
\hline \hline $\begin{array}{l}\text { Number of Market } \\
\text { Participants }\end{array}$ & numSLRs $=\{4,5,6,10,20,50\}$ \\
$\begin{array}{l}\text { Distribution of Users' } \\
\text { Spectrum Valuation }\end{array}$ & $\begin{array}{l}\text { All users have low spectrum valu- } \\
\text { ation and hence, high AT valuation }\end{array}$ \\
Available Spectrum & $\begin{array}{l}\text { numBBUs }=R * n u m S L R s \\
R=\{5,10,15,20,25\}\end{array}$ \\
Market Type & $\begin{array}{l}\text { Band Manager Exchange-based } \\
\text { market }\end{array}$ \\
\hline & TABLE V
\end{tabular}

SPECTRAD RUNNING ENVIRONMENT PARAMETERS

\section{B. Market Viability}

In [2], Band Manager exchange-based markets' viability is determined through the analysis of the following parameters:

\section{Probability of Empty Bid List}

Represents the willingness of users to obtain spectrum from the market. When there is a significant number of empty bid lists, this implies that the users are not interested in acquiring spectrum from the $\mathrm{BM}$ and thus this is an adverse condition for market liquidity.

\section{Probability of Demand Greater than Supply}

When the demand is greater than the supply, the cutoff price is derived from the market activity and finally determined by the highest bidders. Having markets where the demand is not greater than the supply implies that there is not a significant interest in the market for obtaining spectrum and thus, the cutoff price will be the minimum established by the BM.

\section{Average Cutoff Price}

This factor is closely related to the probability of demand being greater than the supply. We desire markets in which the average cutoff price is determined by the bidding activity of the SLRs, and thus well above the minimum cutoff price established by the BM.

\section{Average Number of AT units per spectrum user} In a spectrum trading market, a desirable outcome would portray SUs opting for spectrum rather than AT units. In this manner, we would expect users to keep their AT units' holdings below the average number of ATs that they need to satisfy their average traffic requirements.

\section{Percentage of Assigned Bandwidth Units}

This parameter is directly related to the spectrum efficiency in the market. In this manner, a desirable outcome is for the majority of the BM spectrum assets to be assigned at the end of the bid rounds.

Table VI shows the values that these parameters should have in order to satisfy market liquidity. It should be mentioned that the minimum cutoff price established by the BM is 30 monetary units, condition that justifies the fail value for the average cutoff price criterion. Additionally, note that in the case of perfect fungibility, the fail value for the average number of ATs criterion differs. In perfect circumstances we still shall consider the fail value to be $\geq 10$ as in [2].

We have evaluated the different test scenarios according to these parameters and thus determined the resulting viable markets, which are presented in the following section.

\begin{tabular}{lccc}
\multicolumn{4}{c}{ Viability Criteria } \\
\hline Criteria & $\begin{array}{c}\text { Pass } \\
\text { Value }\end{array}$ & $\begin{array}{c}\text { Fail } \\
\text { Value }\end{array}$ & $\begin{array}{c}\text { Score } \\
\text { Pass/Fail }\end{array}$ \\
\hline P1 - Bid List Empty & $<1 \%$ & $\geq 1 \%$ & $1 /-1$ \\
$\begin{array}{l}\text { P2 - Demand Greater } \\
\text { than Supply }\end{array}$ & $\geq 10 \%$ & $<1 \%$ & $1 /-1$ \\
P3 - Cutoff Price & N/A & $<31$ & $0 /-1$ \\
$\begin{array}{l}\text { P4 - Percentage of } \\
\text { Assigned BBUs }\end{array}$ & $\geq 62 \%$ & $<62 \%$ & $1 /-1$ \\
$\begin{array}{l}\text { P5 - Number of ATs } \\
\text { per User }\end{array}$ & N/A & $\geq 3$ & $0 /-1$ \\
\hline
\end{tabular}

TABLE VI

VIABILITY CRITERIA AND MARKET SCORES

\section{RESUlts}

This section presents the process we have followed in order to determine our market viability 
scores, quantitative measures for the aforementioned scores and the corresponding analysis of these results.

\section{A. Methodology for determining Viability Scores}

During our preliminary analysis, we performed one hundred runs of 5000 time ticks of each of the 30 different combinations of our parameters to obtain the market viability results when incorporating the calculated capacity fungibility score in the price limit. We proceeded to explore how sensitive these viability results were to further decrease of the fungibility score, and thus to lower limit prices. We noted that the results we obtained when performing 20 runs of the model present a difference of \pm 0.06 , in the worst case, with respect to the results we obtained with 100 runs of our model. This difference does not have an impact on the final viability or non-viability condition of a market. In addition, we noticed that regardless of our changes in fungibility considerations, from the perfect fungibility scenario to harsh considerations, the scenarios corresponding to $R \geq 15$ never show viable markets. It is for the two latter reasons that the results we present in this section correspond to the 18 scenarios resulting from the combination of the number of SLRs with $5 \leq R \leq 15$, each of them tested in 20 runs of the model. Thus, we were able to evaluate many more model environments in the available time.

For obtaining the final results, in every run, we recorded the values corresponding to the viability criteria during the last 2000 time ticks. ${ }^{2}$ We obtained an average per run prior to an aggregate average of the 20 model runs for each parameter. Taking into account the pass/fail values for each viability criterion presented in Table VI, we have further determined a set of conditions that account for market liquidity under non-perfect fungibility circumstances.

In our sensitivity analysis results, we present the results under four different fungibility considerations:

- Perfect Fungibility

- Fungibility Score $=0.3448$

- Fungibility Score $=0.25$

- Fungibility Score $=0.15$

\footnotetext{
${ }^{2}$ The first 3000 time ticks are considered a training or warm-up period in the original model and are disregarded for the final score calculations.
}

The first fungibility score corresponds to the capacity probabilistic score (at fixed distance) calculated in the first section of this paper. Considering only this value to portray our fungibility considerations would be an optimistic approach. By so doing, we would infer that the difference in the capacity we would achieve with an alternate frequency is the only concern of the SLR. However, realistically, there are a myriad of factors related to opting for a new frequency. For instance, the radio equipment must be adapted to comply with transmission requirements and there is a learning curve associated with using the new frequency effectively. For consumer facing services (such as mobile telephone services), new handsets have to be sourced, tested and marketed. All these constraints should be reflected in the price the user is willing to pay for the available frequency. To reflect these factors in our analysis, we have tested the two lower fungibility values: 0.25 and 0.15 .

\section{B. Quantitative Results}

After merging all the considerations above in our version of the SPECTRAD model, we can present in figure 4 the resulting market viability scores for every scenario we have tested. It is worth noting that a market will be considered viable when its score is greater than 0 .

To complement the results presented in figure 4, tables VII, VIII, IX and X show the score corresponding to every viability criterion for the tested scenarios with a positive final market score.

Perfect Fungibility

\begin{tabular}{ccccccccc}
\hline \hline & & & & & & & & \\
NumSUs & NumBBUs R & P1 & P2 & P3 & P4 & P5 & Score \\
\hline 6 & 30 & 5 & 1 & 0 & 0 & 1 & -1 & 1 \\
10 & 50 & 5 & 1 & 1 & 0 & 1 & -1 & 2 \\
20 & 100 & 5 & 1 & 1 & 0 & 1 & 0 & 3 \\
50 & 250 & 5 & 1 & 1 & 0 & 1 & 0 & 3 \\
5 & 50 & 10 & 1 & 0 & 0 & 1 & 0 & 2 \\
6 & 60 & 10 & 1 & 0 & 0 & 1 & 0 & 2 \\
10 & 100 & 10 & 1 & 0 & 0 & 1 & 0 & 2 \\
20 & 200 & 10 & 1 & 0 & 0 & 1 & 0 & 2 \\
50 & 500 & 10 & 1 & 0 & 0 & 1 & 0 & 2 \\
\hline
\end{tabular}

TABLE VII

Market Viability SCORE RESUlts for the Perfect FUNGIBILITY SCENARIO 

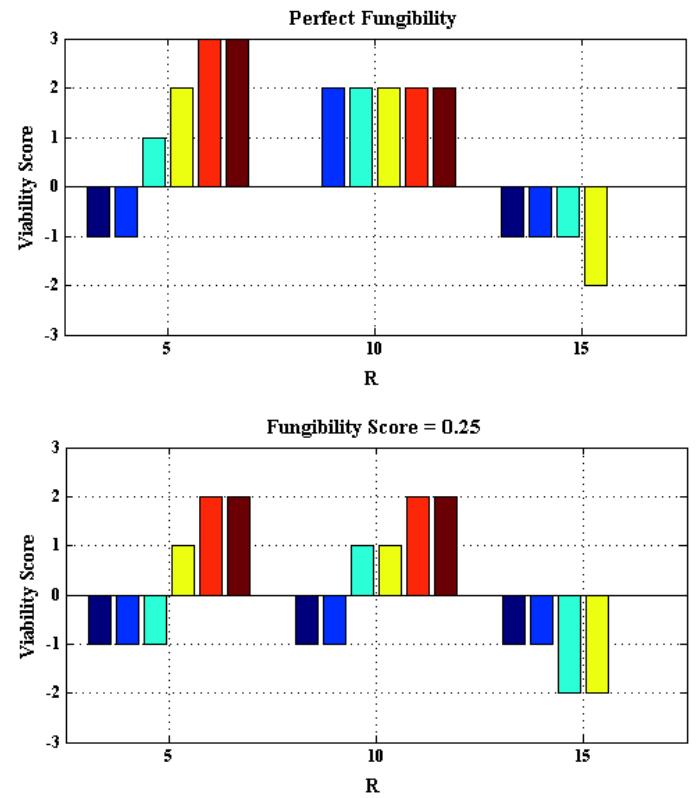
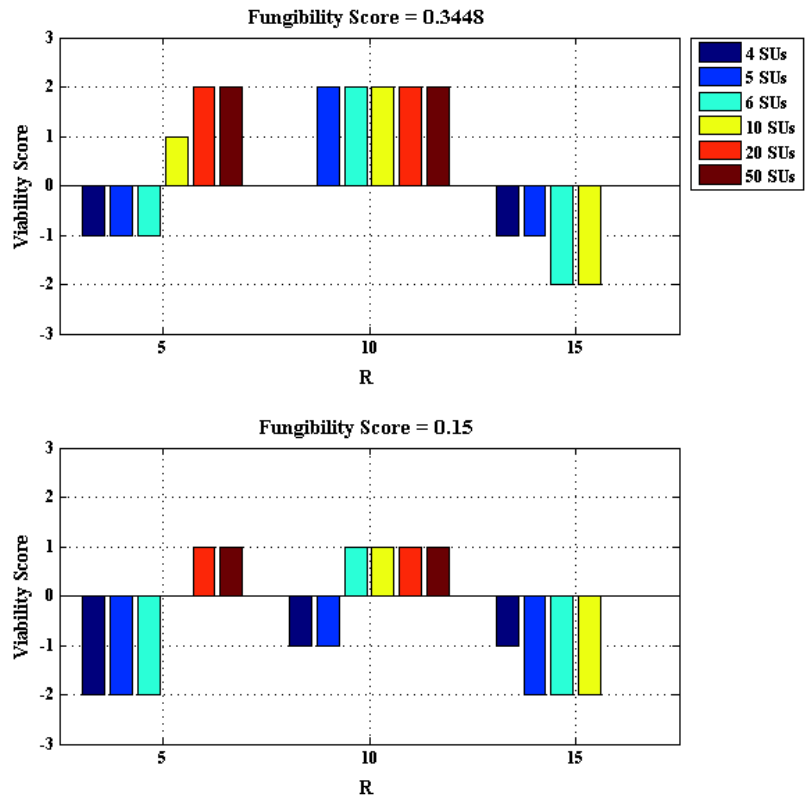

Fig. 4: Market viability scores for different levels of spectrum fungibility.

Fungibility Score equal to Capacity Probabilistic Score

\begin{tabular}{ccccccccc}
\hline \hline & & & & & & & & \\
NumSUs & NumBBUs R & P1 & P2 & P3 & P4 & P5 & Score \\
\hline 10 & 50 & 5 & 1 & 0 & 0 & 1 & -1 & 1 \\
20 & 100 & 5 & 1 & 1 & 0 & 1 & -1 & 2 \\
50 & 250 & 5 & 1 & 1 & 0 & 1 & -1 & 2 \\
5 & 50 & 10 & 1 & 0 & 0 & 1 & 0 & 2 \\
6 & 60 & 10 & 1 & 0 & 0 & 1 & 0 & 2 \\
10 & 100 & 10 & 1 & 0 & 0 & 1 & 0 & 2 \\
20 & 200 & 10 & 1 & 0 & 0 & 1 & 0 & 2 \\
50 & 500 & 10 & 1 & 0 & 0 & 1 & 0 & 2 \\
\hline
\end{tabular}

TABLE VIII

MARKET VIABILITY SCORES OBTAINED FOR CAPACITY PROBABILISTIC FUNGIBILITY SCORE

\section{Analysis}

From the results presented in figure 4, we find market liquidity in all our fungibility scenarios when $5 \leq R \leq 10$. Nevertheless, we do observe some changes as the level of fungibility decreases.

When considering a perfect fungibility environment, with $R=5$, we find market viability when $6 \leq$ num $S U S \leq 50$. When $R=10$, markets begin to be liquid when $n u m S U s \geq 5$. This accounts for a total of 9 viable markets under perfect fungibility conditions when all users have a low valuation of spectrum. As we incorporate our non-perfect fungibility measures, we start losing viable markets. When considering our capacity probabilistic
Fungibility Score equal to 0.25

\begin{tabular}{ccccccccc}
\hline \hline & & & & & & & & \\
NumSUs & NumBBUs R & P1 & P2 & P3 & P4 & P5 & Score \\
\hline 10 & 50 & 5 & 1 & 0 & 0 & 1 & -1 & 1 \\
20 & 100 & 5 & 1 & 1 & 0 & 1 & -1 & 2 \\
50 & 250 & 5 & 1 & 1 & 0 & 1 & -1 & 2 \\
6 & 60 & 10 & 1 & 0 & -1 & 1 & 0 & 1 \\
10 & 100 & 10 & 1 & 0 & -1 & 1 & 0 & 1 \\
20 & 200 & 10 & 1 & 0 & 0 & 1 & 0 & 2 \\
50 & 500 & 10 & 1 & 0 & 0 & 1 & 0 & 2 \\
\hline
\end{tabular}

TABLE IX

MARKET VIABILITY SCORE OBTAINED WHEN THE FUNGIBILITY SCORE IS 0.25

Fungibility Score equal to 0.15

\begin{tabular}{ccccccccc}
\hline \hline & & & & & & & & \\
NumSUs & NumBBUs R & P1 & P2 & P3 & P4 & P5 & Score \\
\hline 20 & 100 & 5 & 1 & 1 & -1 & 1 & -1 & 1 \\
50 & 250 & 5 & 1 & 1 & -1 & 1 & -1 & 1 \\
6 & 60 & 10 & 1 & 0 & -1 & 1 & 0 & 1 \\
10 & 100 & 10 & 1 & 0 & -1 & 1 & 0 & 1 \\
20 & 200 & 10 & 1 & 0 & -1 & 1 & 0 & 1 \\
50 & 500 & 10 & 1 & 0 & -1 & 1 & 0 & 1 \\
\hline
\end{tabular}

TABLE $X$

MARKET ViabiLITY SCORES OBTAINED WHEN THE FUNGIBILITY SCORE IS 0.15

fungibility score (0.3448), when $R=5$, market viability starts when numSUs $\geq 10$. Nonetheless, for $R=10$, the viability conditions are the same as 
those of the perfect fungibility scenario. Under these fungibility circumstances we have 8 viable markets.

When we lower the fungibility score to 0.25 , we now have 7 viable markets as market viability conditions remain for $R=5$ and for $R=10$ viability now starts when $n u m S U s \geq 6$.

In the scenario which considers our final and lowest fungibility score, we loose one more market, for a total of 6 viable markets. In this case, we maintain the same number of viable markets as in the last scenario when $R=10$; however, for $R=5$ viability starts only when numSUS $\geq 20$.

The results included in tables VII to $\mathrm{X}$, permit us to additionally determine the following characteristics associated with market viability:

- All the resulting viable markets have a positive score for the probability of empty bid list criterion. This implies that every successful market has less than $1 \%$ probability of having an empty bid list, which as well represents that users have a high willingness for acquiring spectrum.

- Successful markets have a significant probability of the demand being greater than the supply. The worst case accounts for a probability between $1 \%$ and $10 \%$.

- In our two first fungibility considerations, we can observe that the average value of the cutoff price is above our threshold which gives a score of 0 for this factor. The two following scenarios, where the fungibility score falls to 0.25 and 0.15 , show situations in which the price goes below the "pass" threshold. Nevertheless, it is worth noting that this value is always slightly above the minimum cutoff price as there are always markets where the demand is greater than the supply.

- All the viable markets have a high percentage of spectrum assignment, which shall correspond to at least $62 \%$ of the $\mathrm{BM}$ spectrum holdings.

- In the case of the average number of AT units per spectrum user, we observe negative values for this condition only when $R=5$. As $R$ raises to 10 , which further corresponds to a better level of spectrum supply, the average user will hold less than 3 AT units.

As it can be observed from our results, the number of viable markets is proportional to the impact of the fungibility scores in the limit prices. Besides reducing the number of viable markets, from figure 4 we can also observe that the markets that remain liquid experience decrease in their viability score.

To finalize our evaluation process, we can relate our current results to those presented in [2]. In that work, different spectrum valuation levels were explored, all of them including a combination of low, medium and high spectrum valuation users. Under these circumstances, on average, we have 10 viable markets and we can see that viability is achieved when $5 \leq R \leq 15$. When using the original model for analyzing market viability when only low valuation users are present, and after adapting the viability criteria to the running conditions, we already experience decrease in the viability results. We now will have viable markets when $5 \leq R \leq 10$ for a total of 9 viable markets. We shall remember, still, that for the perfect fungibility environment with only low spectrum valuation users studied in this work, we have not made any modifications to the basis and logic of the model and analysis presented in [2].

\section{CONClusions AND Further WORK}

This work presents an initial approach for merging non-fungibility notions with spectrum trading markets. Our first consideration has been to directly apply our fungibility scores to the spectrum pricing scheme in an spectrum trading environment, such as SPECTRAD. The results obtained depict proportionality between the level of fungibility and the number of resulting viable markets, fact that in a general sense goes along the lines of intuition. What was not expected was that the markets remained fairly robust even in more drastic operational conditions. The markets that were "lost" were ones when spectrum was relatively scarce with respect to demand and where the number of participants was small. Unfortunately, this is consistent with the present mobile carrier environment in most localities, so we do not hold it likely that this form of spectrum market would emerge in practice.

As previously mentioned, this has been an initial approach and our first step towards making a fun- 
gibility impact analysis. Further work shall contemplate new market structures such as the analysis of an exchange-based market without a band manager functionality, in which we can determine additional conditions that may have a significant impact in the viability of a spectrum trading environment. Furthermore, we shall merge additional fungibility considerations that could exploit the multi-dimensional spectrum characteristics and determine more detailed conditions and consequences of fungibility limitations.

\section{REFERENCES}

[1] M. Weiss, P. Krishnamurthy, L. Doyle, and K. Pelechrinis, "When is electromagnetic spectrum fungible?" in IEEE Symposium on New Frontiers in Dynamic Spectrum Access Networks (DySPAN), 2012.

[2] C. E. Caicedo, "Technical architectures and economic conditions for viable spectrum trading markets," July. [Online]. Available: http://d-scholarship.pitt.edu/8510/

[3] R. H. Coase, "The federal communications commission," Journal of Law and Economics, vol. 2, no. ArticleType: research-article / Full publication date: Oct., 1959 / Copyright 1959 The University of Chicago, pp. 1-40, 1959.

[4] J. W. Mayo and S. Wallsten, "Enabling efficient wireless communications: The role of secondary spectrum markets," Information Economics and Policy, vol. 22, no. 1, pp. 61-72, 2010.

[5] M. Bykowsky, "A secondary market for the trading of spectrum: promoting market liquidity," Telecommunications Policy, vol. 27, no. 7, pp. 533-541, 2003.

[6] C. Caicedo and M. B. Weiss, "The viability of spectrum trading markets," IEEE Communications Magazine, vol. 43, no. 3, pp. 46-52, 2011. 